Students from VUW-Te Herenga Waka translate poem 'Consider this' by NZ author Mary Maringikura Campbell into Italian

The poem by Mary Maringikura Campbell, 'Consider this' was first published in the collection 'Maringi' (Kotaha Press, Porirua, 2016) and it has been included in her new book 'Yellow Moon - E Marama Rengarenga' (HeadworX, Wellington, June 2020). Many thanks to the author for granting kind permission to reproduce and translate this poem into Italian and to NEKE for publishing the best translation works of my students of Italian 201-2020, School of Languages and Cultures, Victoria University of Wellington/Te Herenga Waka. My translation of the poem will appear soon also in the selection 'Matariki, sciame di stelle: poetesse maori contemporanee' (Collana 'Affluenti', Edizioni Ensemble, Rome) along with the translation of other poems by Aotearoa's indigenous female authors. Antonella Sarti Evans

\title{
Consider this
}

by Mary Maringikura Campbell

\section{Consider this}

before you pump me with drugs

I am more than a brain

a beating heart

I am skin and bones

a fertile plain

I am my Tūpuna

an ariki

I am yesterday

today and tomorrow

I have a soul

I see...

Tread gently for I have a black dog

who comes and goes

I am a box of recovery tools

a rocky road

I am tapu

inside me is God

my God

the best of me

I am a mother

a daughter

a sister

an auntie

a grandmother

I have two dogs and a cat

and wild birds circling

I am more than just a brain

I am Māori Papa'a

Before you pump me with drugs

consider this, Mr Psychiatrist! 


\section{Consideri questo}

Consideri questo

prima di gonfiarmi di droghe

sono più di un cervello

un cuore che batte

sono carne e ossa

una pianura fertile

sono i miei Tūpuna ${ }^{1}$

sono un'ariki ${ }^{2}$

sono ieri

oggi e domani

ho un'anima

vedo...

Passi dolcemente perché ho un cane nero

che va e viene

sono una scatola di strumenti da recupero

una strada dissestata

sono tapu ${ }^{3}$

dentro di me c'è Dio

il mio Dio

il meglio di me

sono una madre

una figlia

una sorella

una zia

una nonna

ho due cani e un gatto

e uccelli liberi che volano in cerchio

sono molto più di un cervello

sono Papa' $\bar{a}^{4}$ māori

Prima di gonfiarmi di droghe

consideri questo, caro Psichiatra!

Translated by Antonella Sarti Evans

\footnotetext{
${ }^{1}$ Termine di uso comune in inglese neozelandese, significa 'avi, in lingua maori.

${ }^{2}$ Termine di uso comune in inglese neozelandese, significa 'persona di nobili origini', in lingua maori.

${ }^{3}$ Termine di uso comune in inglese neozelandese, significa 'sacro, proibito' in lingua maori.

${ }^{4}$ Un neozelandese di origini miste europee e maori; 'Papa' $\bar{a}$ ' (nella lingua delle Isole Cook) è l'equivalente di

'Pakeha' (in lingua maori) e significa 'bianco'.
} 
Consideri questo

Consideri questo

prima di riempirmi di droghe

sono più di un cervello

un cuore che batte

sono pelle e ossa

una pianura fertile

sono i miei Tüpuna

un ariki

sono ieri

oggi e domani

Ho un'anima

Io vedo...

Sia gentile con me, perché ho un cane nero

che viene e va

Sono una cassetta per gli attrezzi di guarigione

un percorso accidentato

Sono tapu

Dentro di me c'è Dio

il Dio mio

il meglio di me

Sono una madre

una figlia

una sorella

una zia

una nonna

Ho due cani e un gatto

e uccelli selvaggi che volteggiano

Sono più di un cervello

Sono Mãori Papa'a

Prima di riempirmi di droghe

consideri questo, Signor Psichiatra!

Translated by Jessica Goodwin

Tūpuna - Gli antenati

Ariki - Il capotribù

Tapu - Qualcosa di sacro, che può essere anche proibito

Maori Papa'a - Un neozelandese di origini europee e maori delle Isole Cook.

Cane nero - in inglese, il cane nero è un simbolo della malinconia.

Ho scelto di non tradurre le parole in lingua maori direttamente in italiano, perché questi termini hanno un senso che non si potrebbe tradurre in nessun'altra lingua senza perderne la profondità. (Jessica) 
Considera questo

Considera questo

prima di riempirmi di farmaci

Sono più di un cervello

un cuore che batte

Sono pelle e ossa

Una pianura fertile

Sono i miei antenati

Un capo

Io sono ieri

oggi e domani

Ho un'anima

Vedo...

Stai attento perché ho un cane nero

che viene e va

Sono la somma di tutti i vostri tentativi di recupero

una strada dissestata

Sono sacra

dentro di me c'e 'Dio

Il mio Dio

il meglio di me

Sono una madre

una figlia

una sorella

una zia

una nonna

Ho due cani e un gatto

e uccelli selvatici che mi volano intorno

Sono più di un cervello

Sono la figlia di un Māori

Prima che tu mi riempia di farmaci

Considera questo, Signor Psichiatra!

Translated by Phoenix Dingle 
Consideri questo

Consideri questo

prima di imbottirmi di droga

Sono più di un cervello

un cuore che batte

Sono pelle e ossa

una pianura fertile

Sono i miei Tūpuna

un ariki

Sono ieri

oggi e domani

Ho un'anima

Vedo ...

Calpesti gentilmente poiché ho un cane nero che viene e va

Sono una scatola di attrezzi per la guarigione una strada difficoltosa

Sono tapu

dentro di me c'è Dio

il mio Dio

il meglio di me

Sono una madre

figlia

sorella

zia

nonna

Ho due cani e un gatto

e uccelli selvatici che volteggiano

Sono Māori Papa'a

Prima di imbottirmi di droga

Consideri questo, Signor Psichiatra!

Translated by James Marshall 
Considera questo

Considera questo

prima di pomparmi di droghe

Sono più d'un cervello

un cuore pulsante

Sono pelle ed ossa

una pianura fertile

Sono i miei antenati

una capotribù

Io sono ieri

oggi e domani

Ho un'anima

Vedo...

Cammina dolcemente perché ho un cane nero

che va e viene

Sono una scatola di strumenti di recupero

una strada rocciosa

Sono sacra

dentro di me c'è Dio

il mio Dio

il meglio di me

Sono una madre

una figlia

una sorella

una zia

una nonna

Ho due cani e un gatto

e uccelli selvatici che volteggiano

Sono più d'un semplice cervello

Sono Māori (indigena nella terra)

prima di pomparmi di droghe

Considera questo, Signor Psichiatra!

Translated by Leila Foster 
Considera questo

Considera questo

Prima di drogarmi

Sono più di una testa

di un cuore che batte

Sono pelle e ossa

Fertile pianura

Sono il mio Tūpuna ${ }^{5}$

Un $\operatorname{ariki}^{6}$

Sono ieri

Sono oggi e domani

Ho un'anima

Vedo...

Cammina delicatamente perché ho un cane nero

che viene e va

Sono una scatola di strumenti da recupero

Una strada rocciosa

Io sono tapu ${ }^{7}$

dentro di me c'è dio

il mio Dio

La parte migliore di me

Sono una madre

Una figlia

Una zia

Una nonna

Ho due cani e un gatto

E uccelli che volano in cerchio

Sono più di una testa

Sono Maori Papa'a ${ }^{8}$

Prima di drogarmi

Considera questo, Signor psichiatra!

Translated by Georgia Bailey

\footnotetext{
${ }^{5}$ Tūpuna: ancestors: antenati

${ }^{6}$ Ariki: First born child of a first born child, a leader in maori society: Primogenito di un primogenito, capo della società maori

7Tapu: (in this case) a person who has been put into the sphere of the sacred: (Per questo caso) una persona posta nella sfera del sacro.

${ }^{8}$ Maori Papa'a: Cook Islands Maori: maori delle Isole Cook
} 
Considera questo

Considera questo

prima di riempirmi di droghe

non sono solo un cervello

o un cuore pulsante

sono pelle e ossa

una terra fertile

sono i miei Tūpuna

un ariki

sono ieri

oggi e domani

io ho un'anima

vedo...

Fai attenzione perché ho un cane nero

che viene e va

sono una cassetta degli attrezzi da recupero

una strada dissestata

sono tapu

Dio è in me

il mio Dio

il meglio di me

sono una mamma

una figlia

una sorella

una zia

una nonna

ho due cani e un gatto

e uccelli selvatici che volteggiano

non sono solo un cervello

sono Māori Papa'a

Prima di riempirmi di droghe

considera questo, Signor Psichiatra!

Translated by Charlotte Heasman

Tūpuna - gli antenati

Ariki - capo

Tapu - sacro 